\title{
Duality and control theory in spin Josephson junction
}

\author{
Morishige Yoneda ${ }^{1 *}$, Shuji Obata ${ }^{2}$, Masaaki Niwa ${ }^{3}$ and Mitsuya Motohashi ${ }^{3}$ \\ 1 Japan Electronics College, 1-25-4 Hyakunin-cho, Shinjuku-Ku, Tokyo 169-8522, Japan \\ 2 School of Science \& Engineering, Tokyo Denki University, Ishizaka, Hatoyama, Hikigun, Saitama 350-0394, Japan \\ 3 Engineering, Tokyo Denki University, 5 Senju Asahi-cho, Adachi-ku, Tokyo 120-8551, Japan \\ * : Fax: 81-033-363-7685, and/or e-mail: yoneda@jec.ac.jp
}

\begin{abstract}
We developed the theory that the quasi particles is a degree of freedom of a spin magnetic moment, and rebuild the theory into a form suitable for control theory of spintronics. We tried to apply to devices using spin tunneling. Specifically, as the ferromagnetic tunnel junction with a pure spin current, from the analogy of the superconducting Josephson junction, we introduced a model of the ferromagnetic spin Josephson junction and have developed a theory to analyze it.
\end{abstract}

\section{INTRODUCTION}

In various fields of physical systems ${ }^{1,2,3)}$, duality have played a very important role. In particular, in the electric circuit of the electrical engineering, in a relationship between a series circuit and parallel circuit, similar adding laws are satisfied by replace the role of resistance and conductance, inductance and capacitance, current and voltage respectively. Such a rule is known as duality principle of an electrical circuit. As examples of the quantum electronic circuit, Josephson device ${ }^{4,5)}$ in the macroscopic superconducting system is known as a quantum effect device operates by using a quantum flux tunneling. On the other hand, Josephson device in the mesoscopic superconducting system is known as a quantum effect device, ${ }^{6,7)}$ which operates by using the single Cooper-pair tunneling created by a Coulomb blockade ${ }^{8)}$. There is known that Cooper pairs and quantum flux ${ }^{9)}$ are the duality relation to each other ${ }^{10,11)}$. As an analogy for the above example, we have devised a control theory of two types of spin junction devices $^{12,13,14)}$ according to the spin magnetic moments of the magnetic material, which using by duality relation known between the domain wall and the spontaneous magnetization. Our main objective is to build a theory of quantum devices, in which the freedom of a dual particle plays an important role, and we would also like to build upon the duality theory of systems. This paper is composed as follows. In the next section, we describe two types of spin junction device from one another dual relation, and theoretically exhibit the potential for pseudo-spin quantum Hall effect by pure spin current. In Sec 3, as an application of the spin junction mentioned in the previous section, we introduce a single domain wall transistor. In the last section, we present a summary and conclusions.

\section{Duality of the Spin Josephson Junction Model}

As an example of the duality of quantum devices described in the previous chapter, we have devised a control theory of two types of spin junction devices ${ }^{12,13)}$, 14) according to the spin magnetic moments of the magnetic material, which using by duality relation ${ }^{15,16)}$ known between the domain wall and the spontaneous magnetization. One type of these systems, as an analogy for the Josephson junction device, we have devised model of ferromagnetic spin Josephson junction device 3) in the sandwich structure of the FM(ferromagnet) / $\mathrm{AM}$ (antiferromagnet ) / FM junction ${ }^{16,17)}$ that operates using single domain wall tunneling. Another type of these systems, we have devised model of antiferro -magnetic spin Josephson junction device in the sandwich structure of the AM / FM / AM junction ${ }^{16)}$ that operates using single magnetic moment tunneling. Fig.1 shows schematic of FM / AF / FM junction (a) and its equivalent circuit (b).

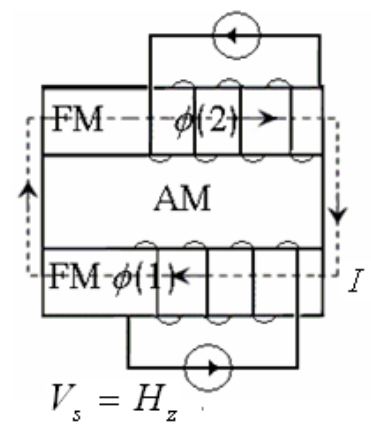

(a)

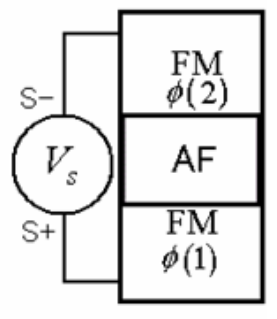

(b)
Fig 1. Schematic of FM / AF / FM junction (a) and its equivalent circuit (b).

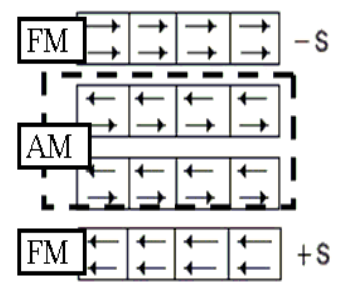

(a)

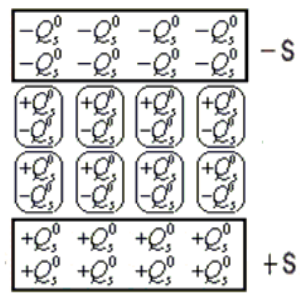

(b)
Fig 2. Conceptual diagram of spin capacitor 
In this case, Antiferromagnetic thin film barrier that sandwiched between the ferromagnetic is functions as a spin capacitor. The Fig.2 (a) and (b) show the conceptual diagram of a spin capacitor ${ }^{16,17)}$. The up spin state" $\rightarrow$ " in Fig. 2 (a) can be considered as the negative spin charge " $-Q_{s}^{0} "$ in Fig.2 (b), and the down spin state" $\leftarrow$ " in Fig. 2 (a) can be considered as the positive spin charge" $+Q_{s}^{0}$ " in Fig.2 (b)

As a model for such ferromagnetic junction systems, first, we consider the Hamiltonian of the XY model with uniaxial anisotropy, as follows:

$$
\begin{aligned}
H_{F M} & =\frac{g \mu_{B}^{0}}{\hbar} \sum_{j} S^{z}(j) H_{z}(j)+J_{z} \sum_{j}\left[S_{z}(j)\right]^{2} \\
& -J_{x y} \sum_{\langle i, j\rangle}\left[S_{x}(i) S_{x}(j)+S_{y}(i) S_{y}(j)\right],
\end{aligned}
$$

The first term is the Zeeman energy due to the $z$ -component of external magnetic field $H_{z}(j)$, where $\mu_{B}^{0}$ is Bohr magneton in $\mathrm{E}-\mathrm{H}$ corresponding, the second term describes the uniaxial anisotropy energy, and the third term describes the XY spin energy. In the Eq. (1), using the relations of $S_{z}(i)=S_{z}^{0} N_{X Y}(i), S_{x}(i)=S_{0} \cos \phi(i)$ and $S_{y}(i)=S_{0} \sin \phi(i)$, we can rewrite the Hamiltonian 16,17) from an analogy of Josephson junctions as follows:

$H_{F M}=Q_{s}^{0} \sum_{j} V_{s}(j) N_{X Y}(j)+E_{Z} \sum_{j}\left[N_{X Y}(j)\right]^{2}-E_{X Y} \sum_{\langle i, j\rangle} \cos [\phi(i)-\phi(j)]$,

where $E_{Z} \equiv J_{z}\left(S_{z}^{0}\right)^{2} \equiv\left(Q_{s}^{0}\right)^{2} / 2 C_{s}$ is spin charging energy per single spin, $Q_{s}^{0} \equiv g \mu_{B}^{0} S_{z}^{0} / \hbar$ is the unit spin charge, $S_{z}^{0} \equiv \hbar / 2 \quad$ is the spin quantum unit, and the spin capacitance $C_{s}$ is defined by $C_{s} \equiv g^{2}\left(\mu_{B}^{0}\right)^{2} / 2 J_{z} \hbar^{2}$, and $E_{X Y} \equiv J_{x y}\left(S_{0}\right)^{2} \equiv I_{c}^{s} \hbar / Q_{s}^{0} \quad$ is XY ferromagnetic spin junction energy per single-spin, $I_{c}^{s} \equiv Q_{s}^{0} E_{X Y} / \hbar$ is critical spin current. $\phi(i)$ and $N_{X Y}(i) \equiv S_{z}(i) / S_{z}^{0} \quad$ represent the relative phase and the relative number in the quasi $\mathrm{XY}$ spin particles, respectively. The approximate commutation relations between $N_{X Y}(i)$ and $\phi(i)$ are given by $\left[\phi(j), N_{X Y}(l)\right] \approx i \delta_{j l}$. Using the Hamiltonian in eq.(1), spin Josephson like equations $\left.{ }^{12}, 13,14,16,17\right)$ are given

$$
\begin{gathered}
V_{s} \equiv \frac{\hbar}{Q_{s}^{0}} \frac{\partial \phi(\mathbf{x}, t)}{\partial t}=\frac{2 N_{X Y}(\mathbf{x}, t)}{Q_{s}^{0}} E_{Z}, \\
I_{s} \equiv Q_{s}^{0} \frac{\partial N_{X Y}(\mathbf{x}, t)}{\partial t}=-I_{s}^{c} \sin \phi(\mathbf{x}, t),
\end{gathered}
$$

where $V_{s}=H_{z}[\mathrm{~A} / \mathrm{m}]$ and $I_{s}[\mathrm{~V} \cdot \mathrm{m}]$ are spin voltage and spin current of FM /AF / FM junction respectively.

Another type of the spin Josephson junction devices has a sandwich structure of antiferromagnet / ferromagnet / antiferromagnet (AF/FM/AF) junction ${ }^{16)}$ (domain wall junctions ).

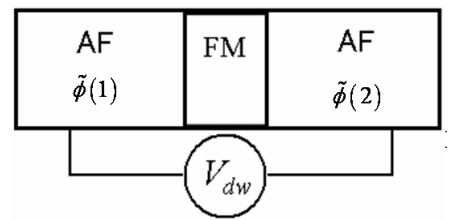

Fig 3. Schematic of AF / FM / AF junction

Fig.3 shows schematic of AF / FM / AF junction . In this case, ferromagnetic thin film barrier that sandwiched between the antiferromagnet is functions as a domain wall capacitor. Where $V_{d w}[V \cdot m]$ is domain wall voltage(dual spin voltage $=$ line of electric force). Fig4 (a) and (b) show the conceptual diagram of a domain wall capacitor. The right-hand thread spin pair state " $\downarrow$ " in Fig. 4(a) can be considered as a negative domain wall charge " $-\Phi_{d w}^{0}=-2 \pi / Q_{s}^{0}[C / m]$ " in Fig. 4 (b) and left-hand thread spin pair state " $\downarrow \uparrow "$ in Fig. 4 (a) can be considered as positive spin charge $"+\Phi_{d w}^{0}=2 \pi / Q_{s}^{0}[\mathrm{C} / \mathrm{m}] " \quad$ in Fig. 4 (b).

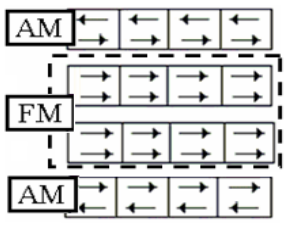

(a)

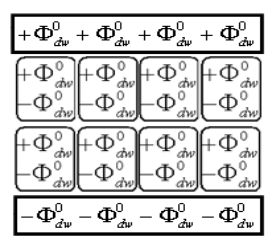

(b)
Fig 4. Conceptual diagram of domain wall capacitor

The Hamiltonian of the domain wall ${ }^{16,17)}$ that is dual to eq.(2) is as follows:

$$
\begin{aligned}
H_{d w}= & \Phi_{d w}^{0} \sum_{i} V_{d w}(i) N_{d w}(i)+2 \pi^{2} E_{x y} \sum_{i}\left[N_{d w}(i)\right]^{2}, \\
& -\frac{E_{z}}{2 \pi^{2}} \sum_{\langle i, j\rangle} \cos [\tilde{\phi}(i)-\tilde{\phi}(j)],
\end{aligned}
$$

where $\tilde{\phi}$ and $N_{d w} \equiv \Phi_{d w} / \Phi_{d w}^{0}$ are phase of the domain wall field and domain wall number, respectively. The approximate commutation relations between $\phi(i)$ and $N_{d w}$ are given by $\left[\tilde{\phi}(j), N_{d w}(l)\right] \approx i \delta_{j l}$. Using the Hamiltonian in eq.(5), equations of the domain wall junctions (dual spin Josephson) ${ }^{16,17)}$ are given

$$
\begin{aligned}
& V_{d w} \equiv \frac{\hbar}{\Phi_{d w}^{0}} \frac{\partial \tilde{\phi}(\mathbf{x}, t)}{\partial t}=I_{s}^{c} 2 \pi N_{d w}(\mathbf{x}, t), \\
& I_{d w} \equiv-\Phi_{d w}^{0} \frac{\partial N_{d w}(\mathbf{x}, t)}{\partial t}=I_{d w}^{c} \sin \tilde{\phi}(\mathbf{x}, t) .
\end{aligned}
$$

where $I_{d w}[\mathrm{~A} / \mathrm{m}]$ and $I_{d v}^{c} \equiv \Phi_{d w}^{0} E_{z} / \pi h[\mathrm{~A} / \mathrm{m}]$ are domain wall current (dual spin current) and critical domain wall current (critical dual spin current), respectively. 
Here, we assume the following duality conditions ${ }^{15,16,17)}$ :

$$
V_{s} \equiv I_{d w}, \quad I_{s} \equiv V_{d w} .
$$

By imposing these duality conditions, we derived the two types of relationships. One is the relationship between the phase of the quasi XY spin particles $\phi$ and the domain wall number $N_{d w}$, and the other type is the relationship between the phase of the domain wall field $\tilde{\phi}$ and the Cooper pair number $N_{X Y}$, which are given as follows:

$$
N_{d w}=\frac{-\sin \phi}{2 \pi}, \quad N_{X Y}=\frac{\sin \tilde{\phi}}{2 \pi} .
$$

From the spin Josephson's like equations (3) and (4), or relations (9), we derived the spin conductance ${ }^{17}$ ) $G_{s}\left[\Omega \cdot m^{2}\right]$ as follows:

$$
G_{s} \equiv \frac{I_{s}}{V_{s}}=G_{Q}^{s} V_{s},
$$

where $G_{Q}^{s} \equiv\left(Q_{s}^{0}\right)^{2} / h$ and $v_{S} \equiv\left(2 \pi^{2} E_{X Y} / E_{Z}\right)\left(N_{d w} / N_{X Y}\right)$ $\equiv N_{X Y} / N_{d w}$ are the quantum spin conductance and Landau level occupation number, respectively. In the case of a condition of $N_{X Y}<<N_{d w}$ or $E_{Z}>>E_{X Y}$, which is the state in a spin insulator. In particular, the extreme case in $G_{s} \rightarrow 0$ is the state in a super spin insulator. In the reverse case, in the condition of $N_{X Y}>>N_{d w}$ or $E_{z}<<E_{X Y}$, which is the state in a spin conductor. In particular, the extreme case in $G_{s} \rightarrow \infty$ is the state in a super spin conductor. As a special case of these conditions $N_{X Y}=N_{d w}$ and $E_{z}=2 \pi^{2} E_{x y}, v_{s}=1$ is given and the spin resistance $G_{s}$ is equal to the quantum spin resistance $G_{Q}^{s}$, which becomes self dual state. If the $\mathrm{n}$ takes an integer value $\left(v_{s}=0, \pm 1, \pm 2 \cdots\right)$, the spin conductance can be considered as pseudo-spin quantum Hall effect ${ }^{16,17)}$ with pure spin current. These results are shown in fig. 5 .

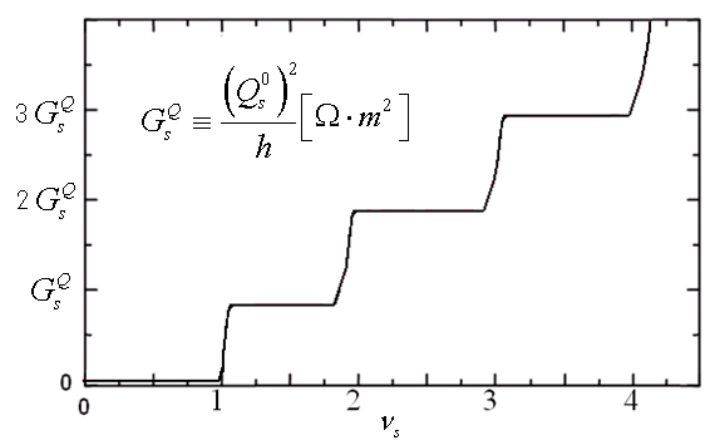

Fig 5 Spin conductance of the pseudo-spin quantum Hall effect with pure spin current

\section{Domain wall blockade}

In this chapter, from the correspondence between the Coulomb blockade ${ }^{18,19,20,21)}$ in electron system and the mesoscopic AF / FM / AF phase junction system, we theoretically derive the conditions under which the domain wall blockade of tunnel flow unit domain wall occurs. From analogy with the single-electron transistor due to the Coulomb blockade, we consider a single domain wall transistor(SDWT) circuit by tunneling of single domain wall charge $\Phi_{d w}^{0} \equiv h / Q_{s}^{0}$ as shown in Fig 6.

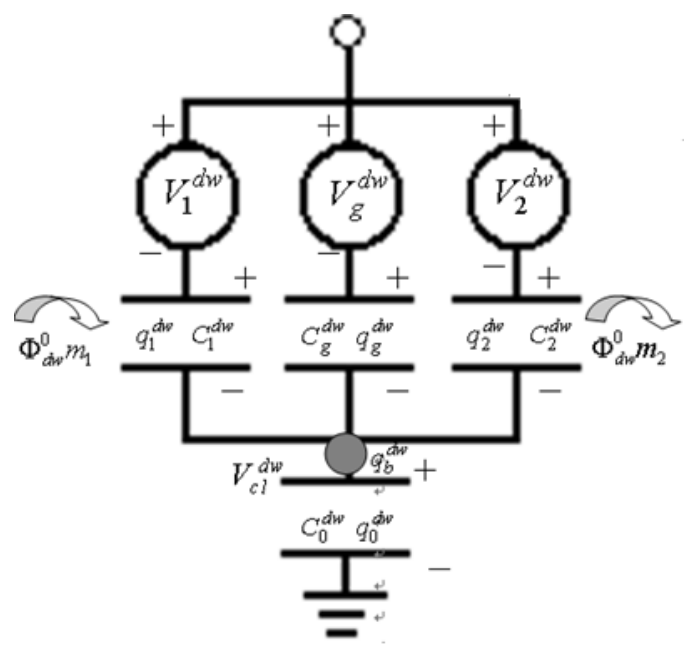

Fig 6 Equivalent circuit of the single domain wall transistor

Here, $q_{i}^{d w}[C / m]$ and $C_{i}^{d w}\left[C^{2} / J \cdot m^{2}\right](\mathrm{i}=0,1,2 . . \mathrm{b}, \mathrm{g})$ are domain wall charge and domain wall capacitance, respectively. In each domain wall capacitor, $m_{1}$ and $m_{2}$ are tunneling number of single domain wall charge in each domain wall capacitor. Free energy of single domain wall transistor in Fig 6 is as follows:

$$
\begin{aligned}
& F\left(m_{1}, m_{2}\right)=\frac{\left[\left(m_{1}-m_{2}\right) \Phi_{d w}^{0}+C_{g}^{d w} V_{g}^{d w}\right]^{2}}{2 C_{\Sigma}^{d w}} \\
& -\frac{m_{1} \Phi_{d w}^{0}}{C_{\Sigma}^{d w}}\left[\left(C_{0}^{d w}+C_{2}^{d w}+C_{g}^{d w}\right) V_{1}^{d w}-C_{2}^{d w} V_{2}^{d w}-C_{g}^{d w} V_{g}^{d w}\right] \\
& +\frac{m_{2} \Phi_{d w}^{0}}{C_{\Sigma}^{d w}}\left[\left(C_{0}^{d w}+C_{1}^{d w}+C_{g}^{d w}\right) V_{2}^{d w}-C_{1}^{d w} V_{1}^{d w}-C_{g}^{d w} V_{g}^{d w}\right] \\
& +\left(\text { Constant term that does not contain } m_{1} \text { and } m_{2}\right),
\end{aligned}
$$

The ground state of this particle number $m_{l}=m_{2}=0$, does not flow to the SDWT (does not occur tunneling of the single domain wall), which is classified into the following four conditions: 

1), $L_{1} \quad \delta F(1,0) \equiv F(1,0)-F(0,0)>0$,
2),$\quad L_{2} \quad \delta F(0,1) \equiv F(0,1)-F(0,0)>0$,
3) , $L_{3} \quad \delta F(-1,0) \equiv F(-1,0)-F(0,0)>0$
4), $L_{4} \quad \delta F(0,-1) \equiv F(0,-1)-F(0,0)>0$,

The cross section of the $V_{2}^{d w}=0$, the condition in which a single domain wall tunneling does not occur, is in the region of the diamond in Fig 7.

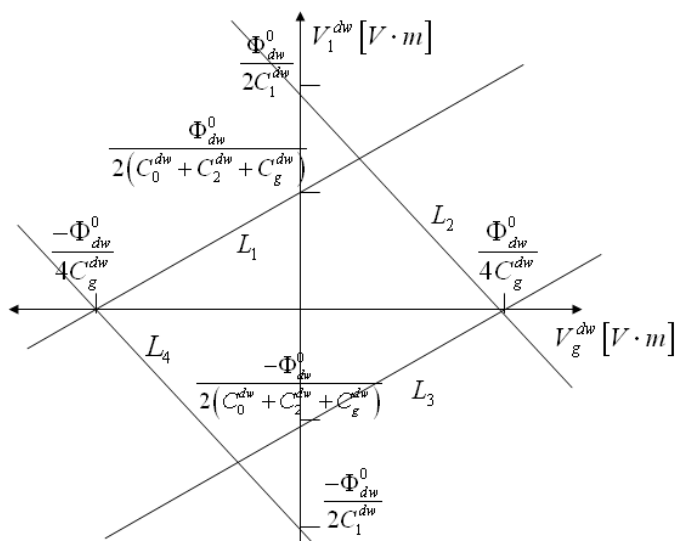

Fig 7 In cross section of the $V_{2}^{d w}=0$, the diamond area single domain wall of tunneling is prohibited.

Further, the cross section of the $V_{g}=0$, in which a single domain wall tunneling does not occur, is in the region of the diamond in Fig 8.

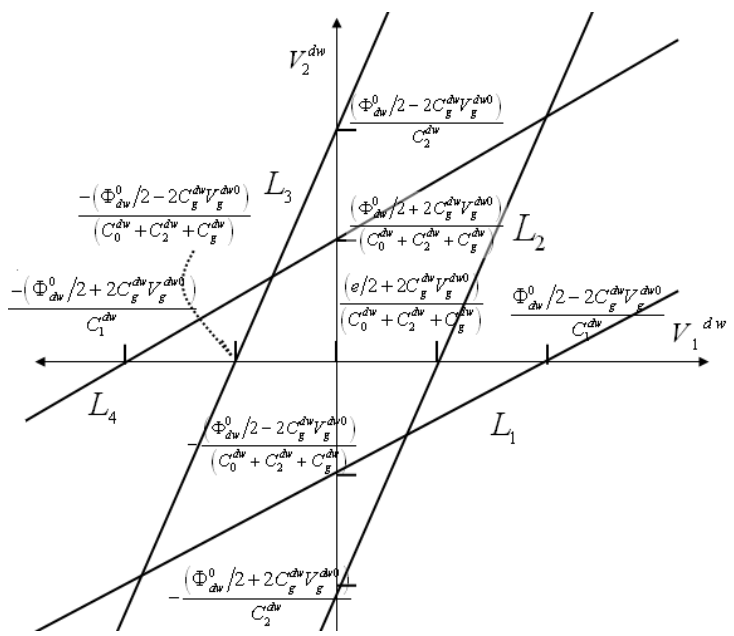

Fig 8 In cross section of the $V_{g}=0$, the diamond area single domain wall of tunneling is prohibited.

\section{Summary and discussion}

In this paper publication, our results are described as follows. It was theoretically shown that there may be the pseudo-integer spin Hall effect with pure spin current As analogy with Coulomb blockade in an electronic system, devised a domain wall Coulomb blockade, from a single domain wall transistor circuit, was determined and conditions of occurrence of single domain wall Coulomb diamond are clarified.

\section{References}

1) H.A.Kramers and G.H.Wannier, Phys.Rev. 60, 252 (1941)

2) R.Savit,Rev.Mod.Phys.,52,453(1980)

3) E.Witten,Phys.Today,50,28(1997)

4) Y.M.Blanter, R.Fazio and G.Sch“on, Nuc.Phy.B Pro.Suppl. V.58,79(1997)

5) R.Fazio and G.Sch"on,Phys.Rev.B,43,5307(1991)

6) M.Y.Choi and J.Choi,Phys.Rev.B,63,212503(2001)

7) M.Y.Choi,B.J.Kim and S.Kim, Phys. Rev. B, 47, 9112(1993)

8) H.R.Zeller and I.Giaver,Phys.Rev.,181,789(1969)

9) M.Yoneda, M,Niwa and S. Shinohara, J.Adv. Sci, Vol.8, No.3 \& 4,180(1996)

(in Japanese)

10) H.Kleinert,GAUGE FIELDS IN CONDENSED MATTER Vol.I (World Scientific,Singapore 1989)

11) H.Kleinert,Lett.Nuovo Cimento,35,405(1982)

12) F. S. Nogueira and K.-H. Bennemann: Europhys. Lett. 67 (2004) 620-626

13) D. Chasse, A.-M. S. Tremblay: Phys. Rev. B 81, 115102 (2010).

14) A. Moor, A. F. Volkov, and K. B. Efetov: Phys. Rev. B 85, 014523 (2012)

15) M. Yoneda, M. Niwa, M. Motohashi. arxiv.org/ cond-mat/1108.3258

16) M. Yoneda, M. Niwa and M. Motohashi: Physica Scripta, Volume T151, (2012).014056

17) M. Yoneda, S. Obata and M. Niwa: Mater. Trans. 55 No 9 (2014) 1510-1512.

18) W. Lu, K. D. Maranowski, and A. J. Rimberg, Phys. Rev. B 65, 060501(R)

19) M.-S. Choi, R. Fazio3, J. Siewert and C. Bruder, Europhys. Lett.53 (2), 251 (2001)

20) D. V. Averin and K. K. Likharev, J. Low Temp. Phys.62, 345 (1986)

21) C. W. J. Beenakker.Phys. Rev. B 44, 1646

(Received December 28, 2014; Accepted February 27, 2015) 